\title{
La Educación Inclusiva como un derecho humano
}

\section{Inclusive Education as a human right}

GUAJARDO-RAMOS, Eliseo ${ }^{1} \uparrow^{*}$, CORRAL-CARTEÑO, Fanny Elizabeth ${ }^{2}$, PADILLA-CASTRO, Laura $^{2}$ y MORENO-AGUIRRE, Alma Janeth ${ }^{2}$

${ }^{I}$ Programa Universitario para la Inclusión Educativa Universidad Autónoma del Estado de Morelos (UAEM)
${ }^{2}$ Facultad de Comunicación Humana,UAEM

ID $1^{\mathrm{er}}$ Autor: Eliseo, Guajardo-Ramos / ORC ID: 0000-0002-1477-0167, CVU CONACYT ID: 265589

ID $1^{\mathrm{er}}$ Coautor: Fanny Elizabeth, Corral-Carteño / ORC ID: 0000-0002-4318-0238, CVU CONACYT ID: 444410

ID $2^{\text {do }}$ Coautor: Laura, Padilla-Castro / ORC ID: 0000-0002-1212-1462, CVU CONACYT ID: 102606

ID $3^{\text {er }}$ Coautor: Alma Janeth, Moreno-Aguirre / ORC ID: 0000-0001-9076-5987, CVU CONACYT ID: 173543

DOI: $10.35429 /$ JPDL.2019.17.5.24.31

Recibido 29 de Agosto, 2019; Aceptado 30 Diciembre, 2019

\section{Resumen}

El interés de muchos estudiosos del campo de la educación por contribuir a la tarea de transitar de una política de Inclusión Educativa hacia una política de Educación Inclusiva involucra aspectos que van más allá del reconocimiento de la problemática que se aborda. Representa en un primer momento identificar y analizar las aportaciones de los expertos que han problematizado sobre los distintos contextos que rodean el tema de la inclusión en la educación desde hace varias décadas. Las propuestas, iniciativas y acciones derivadas de cada uno de los distintos escenarios planteados, tanto por organismos nacionales, como internacionales a lo largo del tiempo han sentado las bases para que la Educación Inclusiva se acerque a su consolidación como un Derecho Humano fundamental. Al mismo tiempo se analiza el impacto de los resultados de una Educación Inclusiva en beneficio de la calidad de vida de quienes a lo largo del tiempo se han identificado como sujetos socialmente vulnerados.

Inclusión, Educación, Derechos Humanos

\begin{abstract}
The interest of many scholars in the field of education to contribute to the task of moving from an educational inclusion policy towards an inclusive education policy involves aspects that go beyond recognizing the issues being addressed. At first, it represents the identification and analysis of the contributions of experts who have problematized on the different contexts surrounding the issue of inclusion in education for several decades. Proposals, initiatives and actions derived from each of the different scenarios mentioned, both by national and international agencies over time, have laid the foundations for inclusive education to approach its consolidation as a fundamental human right. At the same time, the impact of the results of an inclusive education for the benefit of the quality of life of those who have identified themselves as socially violated subjects over time is analized.
\end{abstract}

Inclution, Education, Human right

Citación: GUAJARDO-RAMOS, Eliseo, CORRAL-CARTEÑO, Fanny Elizabeth, PADILLA-CASTRO, Laura y MORENO-AGUIRRE, Alma Janeth. La Educación Inclusiva como un derecho humano. Revista de Filosofía y Cotidianidad. 2019, 5-17: 24-31

\footnotetext{
* Correspondencia del Autor (eliseo@uaem.mx)

$\dagger$ Investigador contribuyendo como primer Autor.
} 


\section{Introducción}

En México la Educación como un Derecho Humano ha sido reconocida en la Constitución a partir de la Reforma llevada a cabo por el Senado de la República el 10 de junio de 2011, no obstante y que la Declaración de los Derechos Humanos en la ONU data de 1948. A nivel internacional el tema de la Educación como Derecho Humano involucra una diversidad de aristas que derivan en una serie de instrumentos que contemplan los Derechos Humanos (DD.HH).

No es posible hablar de una Educación Inclusiva como Derecho Humano sin tocar el tema de la inclusión en educación. Guajardo (2019). refiere como Política gubernamental la Inclusión en Educación enfatizando que la Inclusión Educativa no es sinónimo de Educación Inclusiva por tratarse de dos dimensiones de poblaciones en la educación: la Inclusión Educativa es propia de las estrategias que se siguen en la educación para que las personas con discapacidad "convivan su vida escolar con sus pares sin discapacidad" Duran, L. R. (2019).

El autor destaca que "se trata de reconocer en las personas con discapacidad su condición de persona con todos sus derechos y obligaciones" al ser la educación un derecho humano en todos sus niveles. Por su parte la Educación Inclusiva es definida por la Oficina Internacional de Educación (OIE y IBE, por sus siglas en inglés) de la Organización de las Naciones Unidas para la Educación, la Ciencia y la Cultura, con sus siglas en inglés (UNESCO) con sede en Ginebra, en 2008: "la educación inclusiva: el camino hacia el futuro" definida con todos sus alcances, que por sí misma abarca a toda la diversidad de la población (Guajardo, 2019).

Contemporáneamente se ha enfatizado que la educación es un derecho humano fundamental y un bien público porque gracias a ella nos desarrollamos como personas y como especie contribuyendo al desarrollo de la sociedad. (UNESCO). Para entender cómo ha evolucionado la concepción de la educación como Derecho Humano se propone un recorrido... ...la educación posibilita el ejercicio de otros derechos fundamentales, lo cual supone que no puede ser considerada como un mero servicio o una mercancía negociable, sino como un derecho que el Estado tiene obligación de respetar, asegurar, proteger, promover".... (OREALC, 2007, pp. 27).

\section{La Cronología Internacional de los Derechos Humanos}

Durante la Cumbre Mundial celebrada en el año 2005, los Jefes de Estado subrayaron "el derechos de las personas a vivir en libertad y con dignidad, libres de la pobreza y la desesperación para desarrollar plenamente el potencial humano.

\section{Algunos instrumentos de los DD.HH:}

- La Convención relativa a la lucha contra las discriminaciones en la esfera de la enseñanza. UNESCO, 1960.

- $\quad$ En América Latina en 1969 se aprobó la Convención Americana de los Derechos Humanos y se establece un órgano de protección: la Corte Interamericana de DD.HH. Protege los derechos Civiles y Políticos, más no los Económicos, Sociales y Culturales.

- $\quad$ El Convenio 169 sobre pueblos indígenas y tribales en países independientes, OIT, 1989. Se refiere a su derecho a participar en conocimientos generales para que ayuden en pie de igualdad en la vida de su propia comunidad y en la comunidad nacional (Art. 29); garantizar para ellos una educación en todos sus niveles (Art. 26). Una participación en el diseño de programas educativos con amplia consideración de las lenguas nativas, las tradiciones y la cultura de los pueblos indígenas (Art. 28).

- La Convención Internacional sobre la protección de los derechos de todos los trabajadores migratorios y sus familias, CDTM, 1990. Se trata de la igualdad de oportunidades de los hijos de la población migrante a la educación de los niños nacionales. Así como la libertad de los padres a educarlos de acuerdo a sus convicciones morales y religiosas (Art. 12).

La Declaración de la $44^{\mathrm{a}}$ reunión de la Conferencia Internacional de la Educación, Ginebra, 1994. 
- La Conferencia Mundial sobre Necesidades Educativas Especiales: Acceso y Calidad "Declaración de Salamanca”, UNESCO 1994.

- El Plan de Acción Integrado sobre la Educación para la paz, los DDHH y la Democracia, aprobado en la Conferencia General de la UNESCO en noviembre de 1995.

En 1999 se firmó el Protocolo de San Salvador sobre los derechos económicos, sociales y culturales. La violación de esos derechos se denuncia ante la Comisión de DD.HH., no ante la Corte Interamericana de DD.HH.

Conferencia de Durban contra el racismo, la discriminación, la xenofobia y otras formas conexas de intolerancia (septiembre de 2001).

En el mismo año de la Conferencia Mundial de Durban ocurre la Declaración Universal de la UNESCO sobre la Diversidad Cultural (2001).

Plan latinoamericano para la Promoción de la Educación en Derechos Humanos, Octubre, 2001.

La proclamación de las Naciones Unidas de la Década para la Educación en los DDHH, 1995-2004. Lo que permite que se introduzca en el currículo de todos los países miembros el tema de los DD.HH. Programa Mundial de Educación en Derechos Humanos y el Plan de Acción para la Educación en Derechos Humanos 2005-2009.

Convención sobre los Derechos de las Personas con Discapacidad, CDPD, 2006. -Primer Tratado de DD.HH del Siglo XXI-. Se propone el abordaje de las NNEE.

Los elementos aportados por la Convención sobre los Derechos de las Personas con Discapacidad (CDPD o CRPD, por sus siglas en inglés) es una iniciativa de la Organización de las Naciones Unidas (ONU) que establece "como propósito principal establecer las medidas para que las personas con discapacidad puedan ejercer sus derechos humanos CDPD constituyen un salto cualitativo en la visualización de los derechos humanos para el siglo XXI. En especial en lo que se debe entender por discriminación:
Cualquier distinción, exclusión o restricción por motivos de discapacidad que tenga el propósito o el efecto de obstaculizar o dejar sin efecto el reconocimiento, goce o ejercicio, en igualdad de condiciones, de todos los derechos humanos y libertades fundamentales en los ámbitos político, económico, social, cultural, civil o de otro tipo. Incluye todas las formas de discriminación, entre ellas, la denegación de ajustes razonables" (Art. 2, inciso 3). No obstante el vinculante es CDPD y normativo tratándose de Personas con Discapacidad (Art. 24).

\section{Los DD.HH. en la Educación de México}

Las Convenciones, Declaraciones y Protocolos sobre DD.HH. no tendrían mayor trascendencia si no se educara en el tema. Por eso resulta indispensable desarrollar plenamente el potencial humano, la dignidad y el respeto a los DD.HH. Desarrollar al máximo la personalidad, los talentos y la creatividad. Hacer posible que las personas con discapacidad participen de manera efectiva en una sociedad libre.

Para ello se propone que se lleven a cabo ajustes razonables referidos a las modificaciones y adaptaciones necesarias y adecuadas que no impongan una carga desproporcionada o indebida cuando se requieran en un caso particular para garantizar a las personas con discapacidad el goce o ejercicio en igualdad de condiciones con los demás, de todos los derechos humanos y libertades fundamentales (Art 2, inciso 4).

Así tenemos luego, La Convención de los Derechos del Niño (CDN) que en el año 1989 vino a reafirmar el desarrollo pleno de la personalidad, aptitudes y capacidad mental y física del niño hasta el máximo de sus potencialidades; así como el que se inculque el respeto a los DD.HH., el respeto a la identidad cultural y el respeto al medio ambiente.

Las Convención de los Derechos del Niño (1989); la Convención sobre la eliminación de todas las formas de discriminación contra la mujer (1979), la Convención sobre la eliminación de toda forma de discriminación racial (1969) y la Convención de los Derechos de las Personas con Discapacidad (2006) son derechos universales; porque por el hecho de ser humano se tienen. 
Por ser persona, independientemente de la nacionalidad de que se trate. En cuanto al contenido se refiere a que la seguridad personal, la identidad, la vida y la salud, la educación son Derechos Humanos.

Pasado el Decenio la Oficina del Alto Comisionado para los DD.HH junto con UNESCO, UNICEF, FAO, PNUD y otras agencias de las Naciones Unidas propusieron en el año 2005 el Programa Mundial de Educación en DD.HH, cuya primera fase finalizó en diciembre de 2009. Dicho Programa se encuentra en espera de ser evaluado. Entre las cosas que pretende dicho Programa Mundial se destaca:

Promover la interdependencia, la
indivisibilidad, de los DD.HH
incluyendo los derechos civiles,
políticos, económicos, sociales y
culturales. Así como el derecho al
desarrollo.
El uso de métodos pedagógicos
participativos.
El respeto a la diversidad.

Después de la Declaración Universal de los Derechos Humanos (DUDDHH) la UNESCO aprobó La Convención relativa a la lucha Contra las Discriminaciones en la Esfera de la Enseñanza: CDEE, (1960) fundando la tipificación, la figura de "toda distinción, exclusión, limitación o preferencia fundada en la raza, el color, el sexo, el idioma, la religión y las opiniones políticas o de cualquier otra índole".

Como instrumento vinculante el Pacto Internacional de Derechos Económicos, Sociales y Culturales, el PIDESC (1966) reconoce el derecho de las personas a la educación, la que tendrá por objeto el pleno desarrollo de la personalidad humana y el sentido de su dignidad fortaleciendo el respeto por los derechos humanos y las libertades fundamentales. Promover las actividades de las Naciones Unidas en pro del mantenimiento de la paz.

El citado tratado enfatiza la gratuidad de la enseñanza primaria recomendando que sea progresiva para los niveles de secundaria y superior. Debe favorecerse el acceso a cada uno de los niveles que conforman el sistema educativo.
En análogos términos se encuentra el Protocolo de San Salvador, PSS (OEA, 1988) agregando como otro elemento de diversificación de programas educativos para la formación e instrucción de personas que presentan discapacidad.

La Convención Americana de Derechos Humanos, CADDHH, (1969) y el Pacto Internacional de Derechos Civiles y Políticos, PIDCP (1966) coinciden en cuanto a la libertad religiosa de los padres en la educación de sus hijos.

La Convención de los Derechos del Niño, CDN, 1989. Reafirma el desarrollo pleno de la personalidad, aptitudes, capacidad mental y física del niño hasta el máximo de sus potencialidades; inculcar el respeto a los DD.HH. el respeto a la identidad cultural y al medio ambiente.

La Convención sobre la eliminación de todas las formas de discriminación contra la mujer, CEDAW, 1979. Asegurar la igualdad de derechos entre hombres y mujeres en el ámbito de la educación, la eliminación de todo concepto estereotipado de los papeles masculino y femenino en todos los niveles y todas las formas de enseñanza, mediante el estímulo de la educación mixta (Art. 10).

El Convenio 169 sobre pueblos indígenas y tribales en países independientes, OIT, 1989. Se refiere a su derecho a participar en conocimientos generales para que ayuden en pie de igualdad en la vida de su propia comunidad y en la comunidad nacional (Art. 29); garantizar para ellos una educación en todos sus niveles (Art. 26)...una participación en el diseño de programas educativos con amplia consideración de las lenguas nativas, las tradiciones y la cultura de los pueblos indígenas (Art. 28).

La Convención Internacional sobre la protección de los derechos de todos los trabajadores migratorios y sus familias, CDTM, 1990. Promueve la igualdad de oportunidades de los hijos de la población migrante a la educación de los niños nacionales. Así como la libertad de los padres a educarlos de acuerdo a sus convicciones morales y religiosas (Art. 12). 


\section{Tabla Normativa Internacional:}

Propone como Ejes medulares de la educación, el desarrollo personal y social hasta el máximo de posibilidades, la tolerancia, el pluralismo, la convivencia armónica en sociedad libre Fahd, K., \& Venkatraman, S. (2019, la comprensión, la paz, el respeto a los derechos humanos, las libertades fundamentales y la preservación del medio ambiente: DUDDHH, PIDCP, PIDESC, CADDHH, PSS, CDN, CDEE UNESCO, Convenio 169 OIT, CEDAW, CDTM, CDR y CDPD.

Igualdad ante la ley, Veugelers, W., \& de Groot, I. (2019). igualdad de oportunidades e igualdad de trato. CDEE UNESCO, Convenio 169, CDTM y CDR.

Creencias religiosas. PIDCP, PIDESC, CADDHH, PSS, CONVENIO 169, CDTM y CDR.

Identidad cultural y lingüística, CDR, Convenio 169, CDTM y CDPD.

Inclusión de la diversidad. CDEE, UNESCO, Convenio 169, OIT, CDTM, CDR y CDPD.

Igualdad entre hombres y mujeres en Educación. CEDAW,CDN y CDPD.

Identidad de los niños...CDR y CDPD.

Opinión del educando y ser oído...CDN y CDPD.

Evolución de las facultades de los niños y las niñas CDPD.

No discriminación, incluida la eliminación de prejuicios DUDDHH PIDCP, PIDSC, CADDHH, PSS, CDN, CDEE UNESCO, Convenio 169 OIT, CEDAW, CDTM, CDR y CDPD.

Gratuidad y obligatoriedad DUDDHH, PIDESC, PSS, CDN, CDPD.

Diversificación de programas educativos, PSS.

Realización de ajustes razonables y entrega de apoyo personalizados dentro del sistema regular de educación CDPD.
Participación en el diseño educativo. CONVENIO 169 y CDPD. CDPD.

Educación de Calidad CDEE UNESCO y

\section{Metodología a desarrollar}

El presente artículo se plantea como objetivo principal contribuir al fortalecimiento de una Educación Inclusiva como Derecho Humano fundamental.

Para ello se propone una Investigación bibliohemerografica y documental en tres etapas: En la primera de ellas se analizan las aportaciones de los expertos en el campo de la Inclusión en educación en su tránsito hacia una Educación Inclusiva.

En la segunda etapa se analizan los acuerdos, iniciativas, convenciones y demás instrumentos derivados, tanto de organismos nacionales, como internacionales que han evidenciado la presencia de grupos vulnerados socialmente.

La tercera etapa del presente artículo la constituyen las conclusiones derivadas del análisis de las dos primeras, en donde se pretende mostrar que una Educación Inclusiva representa un Derecho Humano fundamental para mejorar la calidad de vida de la humanidad.

\section{Conclusiones}

Es preciso subrayar que la educación no puede quedar limitada a considerarse sólo como un servicio el que podría ser diferido, pospuesto y hasta negado.

"El Relator Especial sobre el derecho a la educación ha expresado que es fundamental la participación no discriminatoria de una amplia gama de otras entidades (además del estado), para poder hacer realidad la educación inclusiva. En efecto, la educación inclusiva abarca no solo los derechos del alumnado marginado, sino además y de manera más amplia la agilización de los cambios culturales y de valores en el sistema educativo y en la comunidad en general" Informe del Relator Especial sobre el Derecho a la Educación (p. 50). 
Dicha exigibilidad desde una perspectiva jurídica se traducirá en la justiciabilidad en términos de la posibilidad de la persona en contar con acciones judiciales y/o administrativas en caso de violación del derecho a la educación. (pp. 53)

Los Comités de Derechos Humanos de Naciones Unidas abren la posibilidad de realizar comunicaciones (denuncias) por personas $\mathrm{o}$ grupos de personas, cuando se vean afectados en las Convenciones de origen. En ellas se encuentran el derecho a la educación. Una de las condiciones de estas comunicaciones es el agotamiento de los recursos internos, por lo que podemos aseverar que para estos órganos de Tratados, el derecho a la educación es plenamente justificable. Lo propio ocurre en la Comisión Interamericana de Derechos Humanos, en el ámbito regional. (p. 53). En definitiva, la justiciabilidad podría contribuir a subrayar el contenido y sustancia del derecho pleno a la educación, con el significativo impacto en las personas individualmente consideradas en el sistema educativo global, como también en la solidez de un Estado de derecho pleno". (p. 53)

Los Estados, como partes de los tratados de los DD.HH, tienen la obligación de respetar, proteger y satisfacer el derecho a la educación con independencia de las condiciones de emergencia. Además, cada persona es sujeto del derecho a la educación, con independencia de su estatus jurídico particular, sea el de refugiado, niño soldado o desplazado interno. De modo que aunque cada persona comparta el mismo derecho a la educación, pocos individuos comparten las mismas necesidades educativas.

Por otro lado, los Estados tienen la obligación jurídica principal de asegurar la educación, incluso si carecen de la capacidad indispensable para hacerlo. Por esta razón debe entenderse que los compromisos legales de la comunidad internacional han sido concebidos para responder a las necesidades integrales de las personas, de manera que esos compromisos incluyen el suministro de asistencia educativa, tal como lo contempla el Art. 28.3 de la Convención de los Derechos del Niño. (p. 65) En 1993, ACNUR adoptó su política sobre niñas y niños refugiados, que incluye el principio rector de que en todas las acciones referentes a los niños y las niñas refugiadas debe brindarse consideración primaria a su interés superior.
El Convenio IV de Ginebra relativo a la protección debida a las personas civiles en tiempos de guerra establece que deben tomarse medidas para asegurar que los niños huérfanos o separados de sus familias como resultado del conflicto tengan acceso a la educación. (ver art. 50 del Convenio, referente a las obligaciones de la potencia ocupante; y el artículo 78 del Protocolo adicional I referente a la protección del derecho a la educación en evacuaciones).

El Protocolo adicional II a los Convenios de Ginebra de 1977 se aplica a los conflictos no internacionales y por lo tanto es de la mayor relevancia actual, pues abarca las acciones de grupos armados no estatales y afirma en su artículo 4 (párr. 3 inciso a) la obligación de dar a los niños y a las niñas el cuidado y ayuda que requieran, incluyendo el derecho a recibir educación.

De importancia particular es el artículo 8 del Estatuto de Roma de la Corte Penal Internacional, que confirma que todos los ataques internacionales contra los edificios destinados a la educación constituyen crímenes de guerra y por lo tanto se encuentran sujetos a la jurisdicción del Tribunal (Art. 8 Párr. 2, inciso e), iv).

El Consejo de Seguridad en 1999 aprobó la resolución 1261 (1999) que condenaba todos los ataques contra los "objetos protegidos en virtud del derecho internacional" incluidas las escuelas y exhortaba a todas las partes interesadas a que pusieran fin a tales prácticas.

En el marco de acción de Dakar, en el que se indica que "ningún país que se comprometa seriamente con la educación para todos se verá frustrado por falta de recursos en su empeño por lograr esa meta". La implicación es clara: cualquier Estado dispuesto, pero incapaz de asegurar la educación primaria debería poder obtener los fondos indispensables para ese fin.

La educación es un tema de desarrollo antes que una actividad humanitaria $\mathrm{y}$, menos aún, un Derecho Humano. Al obrar así se ha fallado en la responsabilidad contenida en los instrumentos internacionales que definen la naturaleza y el contenido del derecho a la educación. 
Con la creación del Grupo Integrado de Educación del Grupo de Trabajo del comité Permanente entre Organismos, la educación como prioridad en la ayuda humanitaria seguirá estando fuera hasta que no sea reconocida por todos los Estados miembros. Las prioridades de los donantes han pasado de financiar necesidades de desarrollo de largo plazo a concentrarse en el alivio de la ayuda humanitaria de desastres, en actividades tradicionales de alimento, salud y abrigo.

No existe una agencia única que se ocupe de la ayuda humanitaria para la educación. La educación de emergencia es liderada por la UNESCO en combinación con ACNUR y UNICEF. Uno con refugiados y el otro con niños, niñas y adolescentes.

La calidad de la educación implica una responsabilidad colectiva que incluye el respeto a la individualidad de todas las personas; implica el respeto y la potenciación de la diversidad, en la medida en que todo aprendizaje exige el reconocimiento del otro como legítimo otro. Por esta razón, la búsqueda de los consensos y el reconocimiento de las diferencias constituyen las fuentes educativas más importantes en la construcción de la cultura de la paz. El desarrollo curricular y el amplio espectro de actividades pedagógicas requieren de actitudes democráticas y participativas por parte de maestros y estudiantes. Éstas deben incluir a todos los sectores comunitarios y muy especialmente a los grupos históricamente marginados.

Esta investigación aporta el llevar más allá de la Educación como un Derecho Humano, sino considerara a la misma Educación Inclusiva como un Derecho Humano. Lo cual garantiza y tutela también la estrategia principal para acceder verdaderamene a la Educación en las condiciones que aseguren el derecho al aprendizaje, no solo a la enseñanza a la que tengan a bien exponer a la persona sin que se preocupen si está o no accediendo al conocimiento.

\section{Agradecimiento}

Agradecimiento a la Universidad Autónoma del Estado de Morelos y al Cuerpo Académico Estudios Transdiscilinarios sobre Grupos Vulnerables (UAEMOR-CA-142) por el apoyo brindado.

\section{Referencias}

Ainscow, M (1999) Desarrollo de escuelas inclusivas. Madrid: Narcea, 2001.

Booth, T.\& Ainscow, M. (2000) Index for inclusión .Guía para la evaluación y mejora de la educación inclusiva.. Madrid: Consorcio Universitario para la Educación Inclusiva. (consorcio.educacioninclusiva@uam.es)

Blanco Guijarro, Rosa, Presentación, Revista latinoamericana de educación Inclusiva; Facultad de Ciencias de la Educación; Septiembre 2010/Volumen 4/No 2.

Carbonell i París, F. (2000) Decálogo para una educación intercultural. Cuadernos de Pedagogía, 290, 90-94 Coll, C. (1994) “El tractament de la diversitat a la LOGSE" Espais Didactics, 7, 14-22

Coll, C. \& Miras, M. (2001) Diferencias individuales y atención a la diversidad en el aprendizaje escolar. En C. Coll, J. Palacios A. Marchesi, (Comp) Desarrollo psicológico y educación, Vo.2. Madrid: Alianza

Cisternas Reyes, M.S. (2010) Derecho a la Educación: marco jurídico y justiciabilidad en: Revista latinoamericana de educación Inclusiva; Facultad de Ciencias de la Educación; 4(2), 4157.

Duran, L. R. (2019). Rethinking an inclusive model of education from complex thinking for the teaching of human rights. Política, Globalidad y Ciudadanía, 4(8), 20.

Fahd, K., \& Venkatraman, S. (2019). Racial Inclusion in Education: An Australian Context. Economies, 7(2), 27.

Echeita, Gerardo. Perspectivas y dimensiones críticas en las políticas de atención a la diversidad. Publicado en Alambique, Didáctica de las Ciencias Experimentales, 44. 7-16(2005).

Gardner, H. (1995). Inteligencias múltiples: la teoría en la práctica. Barcelona: Paidós

Gimeno, J. (2001) Políticas de la diversidad para una educación democrática igualitaria. En Sipán, A. (Coord.) Educar para la diversidad en el siglo XXI. Zaragoza: Mira editores. 
Guajardo, E. (agosto 20 de 2019) recuperado de https://elregional.cm.mx/Inclusión-educativa-en la-uaem-educación-inclusiva-uaem

Hevia- Rivas, R. (2010) El Derecho a la Educación y la Educación en Derechos Humanos en el contexto internacional, en: Revista latinoamericana de educación Inclusiva; Facultad de Ciencias de la Educación; 4 (2), 25-39

Muñoz, V. (2010) El Derecho a la Educación en situaciones de emergencia, en: Revista latinoamericana de educación Inclusiva; Facultad de Ciencias de la Educación;4(2), 5977

OEA. (1999) Convención Interamericana para la Eliminación de Todas las Formas de Discriminación contra las Personas Portadoras de Deficiencia (Declaración de Guatemala).

Parra-Dussan, C. (2019). Convención Sobre los Derechos de las Personas con Discapacidad: antecedentes y sus nuevos enfoques, 16 International law, Revista Colombiana de Derecho Internacional, 347-380.

Veugelers, W., \& de Groot, I. (2019). theory and Practice of Citizenship education. In Education for Democratic Intercultural Citizenship (pp. 1441). Brill Sense.

Zembylas, M. (2019). A Butlerian perspective on inclusion: the importance of embodied ethics, recognition and relationality in inclusive education. Cambridge Journal of Education 